\title{
Blood Platelet's Behavior on Nanostructured Superhydrophobic Surface
}

\author{
Ming Zhou ${ }^{1, a}$, Jiahong Yang ${ }^{1}$, Xia Ye ${ }^{1,2}$, Aoran Zheng ${ }^{1}$, Gang $\mathrm{Li}^{1}$, Peifang \\ Yang $^{3}$, Yi Zhu ${ }^{4}$ and Lan Cai ${ }^{1}$ \\ ${ }^{1}$ Center for Photonics Fabrication, Jiangsu University, Zhenjiang 212013, PR China \\ ${ }^{2}$ School of Mechanical Engineering, Jiangsu Teachers University of Technology, Changzhou, PR \\ China \\ ${ }^{3}$ Department of Obstetrics, Jiangbin Hospital, Zhenjiang 212001, PR China \\ ${ }^{4}$ Department of Cardiology, Huadong Hospital, Shanghai 200040, PR China \\ azm_laser@126.com (Corresponding author).
}

received paper, 08.12.2007 received revised paper,05.01.2008 accepted date, 05.01.2008

\section{Keywords: Soft Lithography, Textured Structure, Surperhydrophobic, Platelet Adhesion}

\begin{abstract}
Regular arrays of micro-pillars and nano-grooves structures on the silicon wafer are fabricated by using soft lithography, and the three dimension morphology of textured surface is observed by using scanning electron microscopy (SEM) and atomic force microscope (AFM). The static water contact angles are measured by using contact angle meter to characterize the wettabilities of these surfaces. To investigate how the presence of topography and the variations of wettability affect the haemocompatibility of textured surface contacted with blood, different patterned surfaces are designed and fabricated, and blood platelet adhesion test is carried out on these surfaces. The adhesion and coagulation of platelets are inspected by scanning electron microscopy (SEM). Experimental data presented in this paper indicate that different surface roughness and wettability are the important factors for blood platelet adhesion. The amount of adsorbed blood platelet is low on textured surfaces, compared with that on the flat surface. Especially, there is no coagulation and activation on the surface with nanometer grooves. That is to say, the superhydrophobic surface is apt to decrease blood platelet adhesion. The study suggests that surface with suitable wettabililty and textured structures exhibits superior blood compatibility.
\end{abstract}

\section{Introduction}

When biomaterial surface gets into contact with fresh blood, the blood proteins will be adsorbed onto it rapidly, followed by such activities as clotting factors activation, platelet adhesion and activation. Finally, the thrombus is formed on artificial biomaterial surfaces[1-5]. For the implants, it is important to prevent blood platelet from adhering to replant surface. The lack of adhesion is of potentially great importance in medical treatment of replants. There are many cases in which it is desirable to make a non-adhesion surface: the replacement tube used in vascular repair is a good example. Therefore, in order to improve the blood compatibility of biomaterial, various techniques such as plasma deposition, self assembling and laser irradiation, [1-5] have been applied to modify the surface for preventing thrombus formation. The cues with which the biomaterial contact in vivo can be chemical or purely physical related to the surface topography. It has been thought that the interaction between the surface of the artificial biomaterial and biological environment is a key 
factor to determine the biocompatibility of materials. The relationship between the physicochemical state of surface and blood compatibility of the materials is of topical concern[6]. Various studies have shown that the interfacial interactions are related to surface properties such as surface chemical composition, surface wettability and surface roughness[7-9]. Our study is undertaken to examine how arrays of pillars/grooves surface and wettability affect platelet adhesion.

Varieties of patterned structures were fabricated by soft lithography. It is proved that soft lithography is a both simple and inexpensive approach to pattern features on multiple length scales (from nanometers to centimeters). With this technique, highly ordered and orientated surfaces can be prepared to study cell-biomaterial interaction and has drawn much interest recently[10-12]. In this study, textured surfaces with arrays of micro-pillars/nano-grooves were fabricated by using soft lithography. To evaluate the fidelity of surface, the three dimension morphology was observed by using atomic force microscope (AFM) and scanning electron microscopy (SEM). Surfaces with different textured structures could cause water-repellency, and were considered to be anti-adhesive[13]. The surface hydrophobicity was responsible for less protein adsorption and less cellular adhesion, resulting in improved biocompatibility[14-15]. In addition, the wettability of these surfaces which was measured by contact angle meter was related to the result of platelet adhesion. Blood platelet adhesion test was carried out to estimate blood compatibility, with a view to the influential factors of surface roughness and wettabililty. The adhesion and coagulation of platelets were demonstrated by SEM.

\section{Materials and methods}

Surface preparation. In order to obtain the patterned mold, micro-pillar and nano-groove structures on the base of different silicon wafer surfaces are fabricated using electron beam lithography. After mold patterning, the patterned mold were exposed to Trichloro(1H,1H,2H,2H-perfluorooctyl)silane $\left(\mathrm{CF}_{3}\left(\mathrm{CF}_{2}\right)_{5} \mathrm{CH}_{2} \mathrm{CH}_{2} \mathrm{SiCl}_{3}\right.$, Aldrich) for $2 \mathrm{~h}$ in a low-pressure chamber, allowing an easy removal of the polydimethylsiloxane(PDMS) membrane from the wafer mold. PDMS gel (Sylgard 184, Dow Corning Co.) is made of elastomer and catalyst in the ratio of 10:1. Then we spread the gel over the patterned silicon mold surface gently. Then it is degassed at a vacuum chamber for $30 \mathrm{~min}$, and cured at $100{ }^{\circ} \mathrm{C}$ for $60 \mathrm{~min}$ before peeling PDMS membrane off the silicon mold. The prepared surfaces were observed by using the scanning electron microscopy (SEM, JSM-840A, JEOL LTD., Japan) and the atomic force microscopy (AFM, Explorer EX300500, Veeco, USA).

Contact angle determination. Contact angles method was used to determine the wettability of arrays of patterned surfaces. We determined the static contact angle(CA) of water by the sessile drop method with a contact angle meter (OCAH200, Dataphysics, Germany) and average five measurements of water were used on each sample surface to get desirable statistical results. We used $1-\mu \mathrm{L}$ drop for every experiment.

Platelet adhesion experiment. Fresh blood added with anticoagulant citrate dextrose (9:1) was centrifuged at $1000 \mathrm{rpm}$ for 10min to obtain platelet-rich plasma (PRP). Patterned PDMS surfaces and smooth PDMS surface which is used to show a contrast were cut into pieces of $10 \times 10 \mathrm{~mm}$. After equilibrated in Phosphate Buffer Solution (PBS, PH 7.4) for 120min, these pieces of PDMS surfaces were incubated in a fresh human PRP at $37{ }^{\circ} \mathrm{C}$ for $60 \mathrm{~min}$. And then, the samples were rinsed three times with PBS to remove the weakly adsorbed platelet. After rinsing, PBS (PH 7.4) containing $2.5 \mathrm{vol} \%$ glutaraldehyde was introduced to fix the platelets adhered to surface, and then dehydrated by being treated with gradual ethanol/distilled water mixtures from $40 \%$ to $100 \%$ ( $10 \%$ ethanol increment) at $4{ }^{\circ} \mathrm{C}$ for $30 \mathrm{~min}$. After drying at room temperature, the samples were 
sputtered with gold and examined using SEM. Results of SEM experiments were shown with representative micrographs. Five fields of view were chosen randomly to obtain good statistical density of the platelet adhesion. The flat PDMS sample was taken as a contrast. The final number of platelets adhering to the surface was expressed as a percentage of the control.

\section{Results and discussions}

Characterization of surface. The geometry size of square pillars arranged in a regular array on the silicon wafer surface is as follows: width a, spacing $b$ and height $h$, as is sketched in figure 1 . In this experiment, the size of a is $15 \mu \mathrm{m}, \mathrm{h}$ is $5 \mu \mathrm{m}$ and spacing b gradually increases from $5 \mu \mathrm{m}$ to $60 \mu \mathrm{m}$ (5 $\mu \mathrm{m}$ increment). A SEM image of the part of the whole micro-pillars of the PDMS surface is shown in figure 2. It can be evidently seen from the observed SEM images that the surface quality of the PDMS surface with micro-pillar array structures is very good .There aren't almost surface deficiency and damnification on the surface. The aspect ratio is also perfectly vertically. The size of the top and the bottom of the pillars are all about $15 \mu \mathrm{m}$. It indicates that soft lithography has better fidelity. The silicon mold and the PDMS surfaces with nano-grooves fabricated by using soft lithography are examined by the AFM. The results are displayed in figure 3. It shows that these textured microstructures are evenly distributed and the surface roughness is almost identical with the mold. And the PDMS replica has the grooves with an average depth of 130nm. However, the depth is less than that of the mold which is $200 \mathrm{~nm}$. The disfigurement is primarily resulted from that PDMS did not penetrate into silicon mold, which was related to factors of degassing time, mold cleanness and curing temperature. It demands further amelioration in our laboratory.

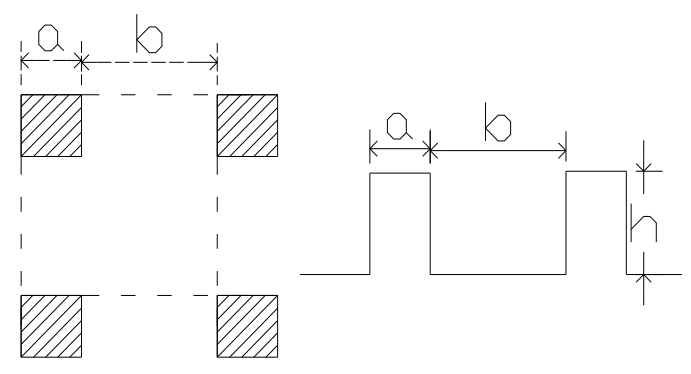

Fig.1 Top and cross-section views of roughness geometry of square pillars $(\mathrm{a}=10 \mu \mathrm{m}, \mathrm{h}=5 \mu \mathrm{m}$, $\mathrm{b}=5 \mu \mathrm{m} \sim 60 \mu \mathrm{m}, 5 \mu \mathrm{m}$ increment).
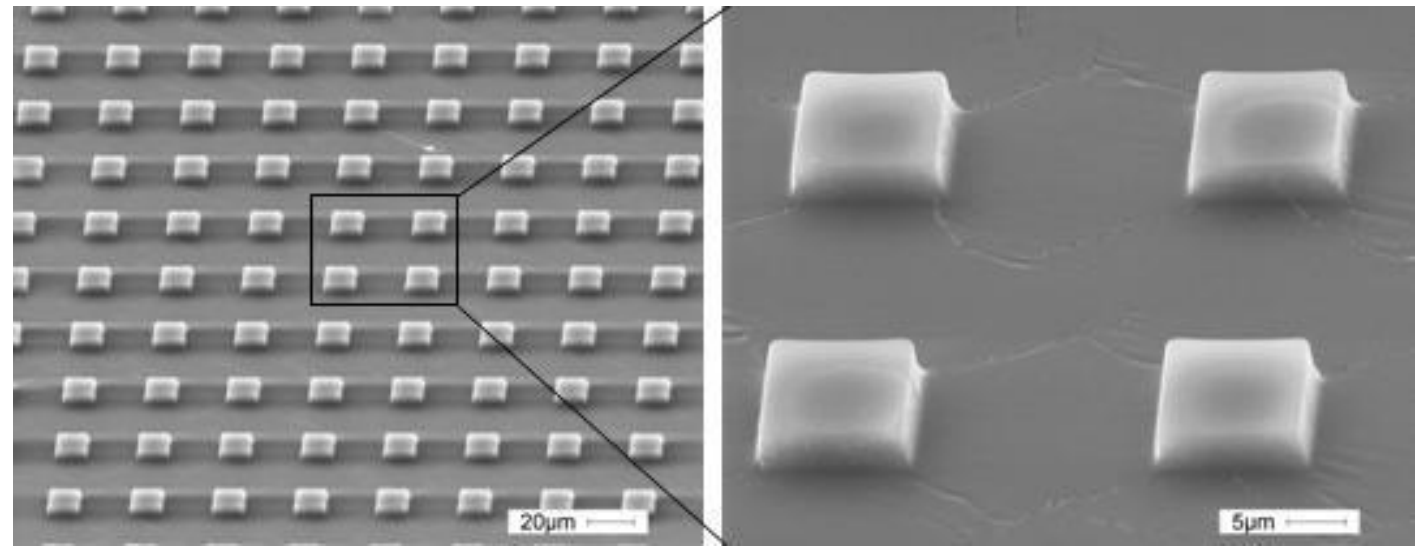

Fig. 2 SEM image of arrays of pillar structures made of PDMS.

Contact angle measurement. To compare the wettability of PDMS replica with different roughness, the water contact angles were measured. Figure 4 shows the relationship between contact angle and the spacing $b$ of the micro-pillars on the PDMS surfaces. The data points at zero 
were obtained on flat PDMS surface. The contact angle increases by $40^{\circ}$ on microstructure surface compared with that on flat surface. The micro-pillars surface gives the largest contact angle of $156^{\circ}$ and small roll-off angle of $2^{\circ}$ at spacing $25 \mu \mathrm{m}$. In addition, according to the measurement the contact angle of the water drop of the PDMS surface with nano-grooves is only about $120^{\circ}$. Therefore, we can say that the contact angles varied significantly due to surface pattern features, although the chemical compositions of PDMS samples are the same.

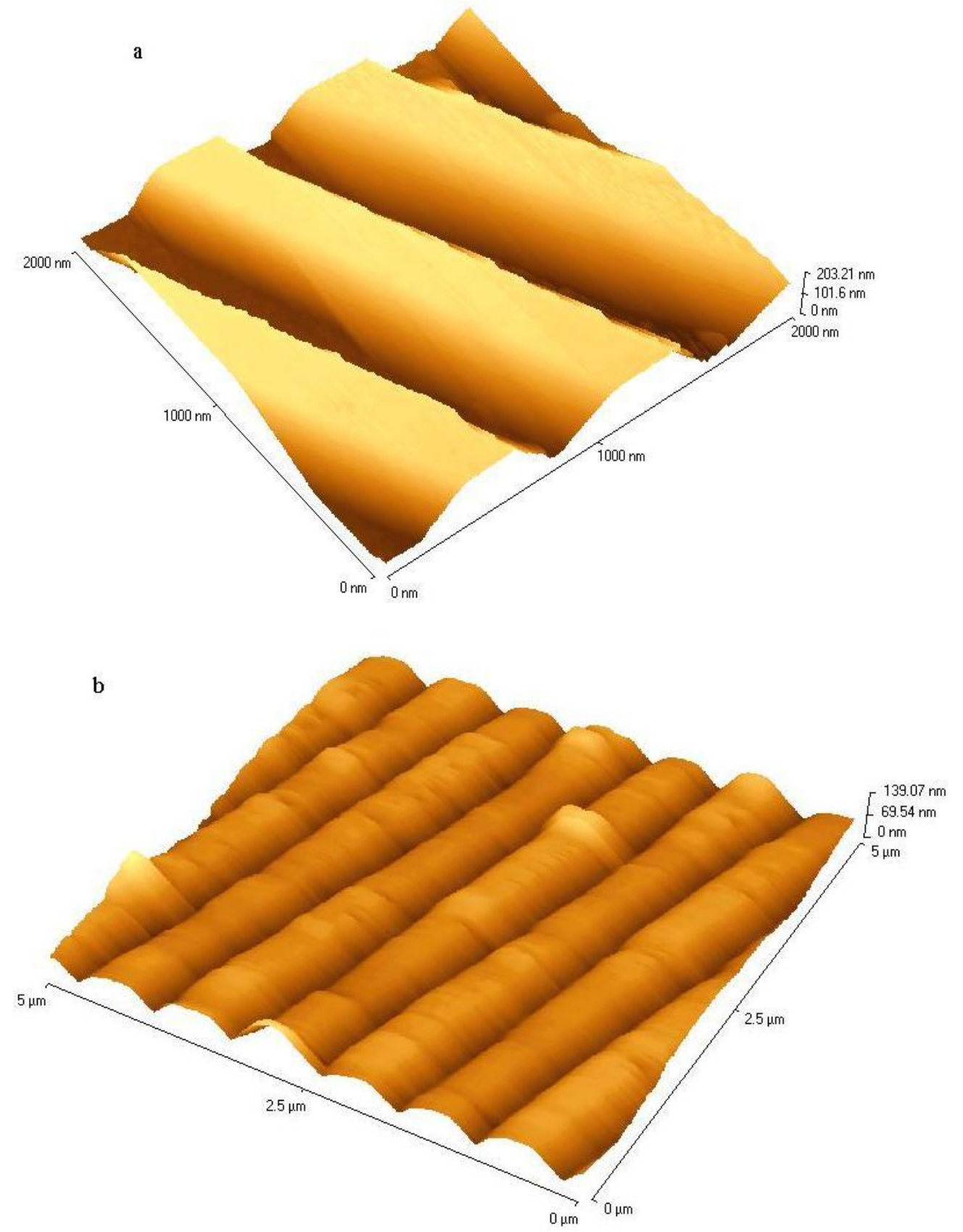

Fig. 3 AFM images of (a) silicon mold and (b) PDMS sub-micro patterned by replica molding.

Platelets adhesion. Platelet-surface interaction is a very complex and dynamic series of events. Platelet adhesion on the surface is invariably followed by the appearance of platelet aggregates and platelets spreading and subsequent thrombus formation that causes the potential danger of using artificial materials in vivo[16]. Platelet adhesion and activation are the indicators of thrombosis on a biomaterial surface. Utilizing the platelet adhesion test in vitro, we evaluate the effect of surface wettabililty and textured fabrics on the blood compatibility. The number of adhered platelets on the surface was counted and expressed as a percentage of the control showed in figure 5. And the 
spacing $b$ of the micro-pillars of the samples which used to in this experiment are $25 \mu \mathrm{m}, 35 \mu \mathrm{m}, 45$ $\mu \mathrm{m}$ respectively. The platelet adhesion displayed significant difference in the behavior of platelet adhesion among different textured surfaces.

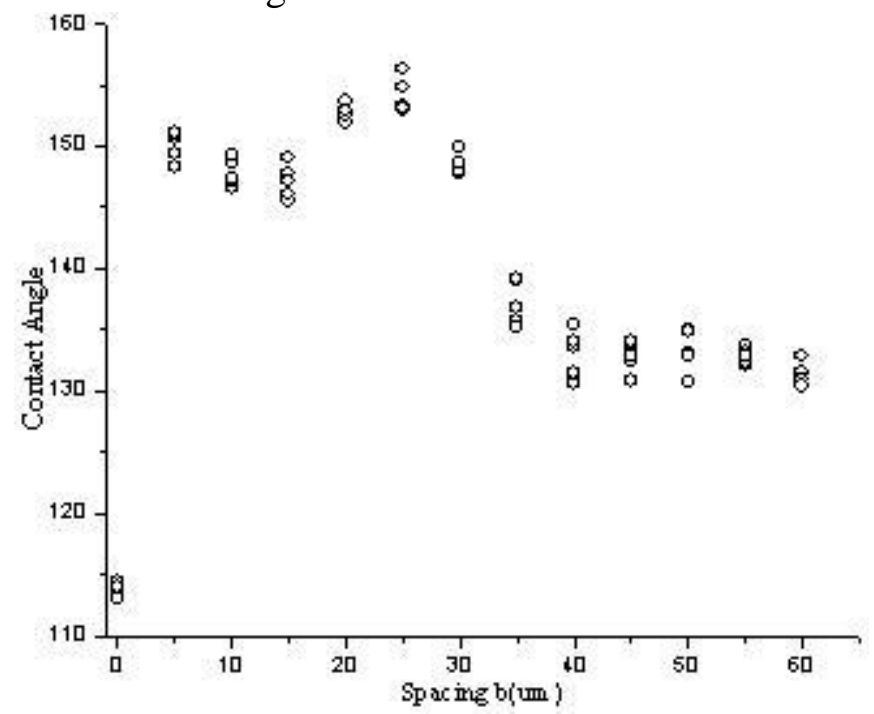

Fig. 4 Static contact angles on arrays of micro-pillars when spacing $\mathrm{b}$ is varied from $5 \mu \mathrm{m}$ to $60 \mu \mathrm{m}$.

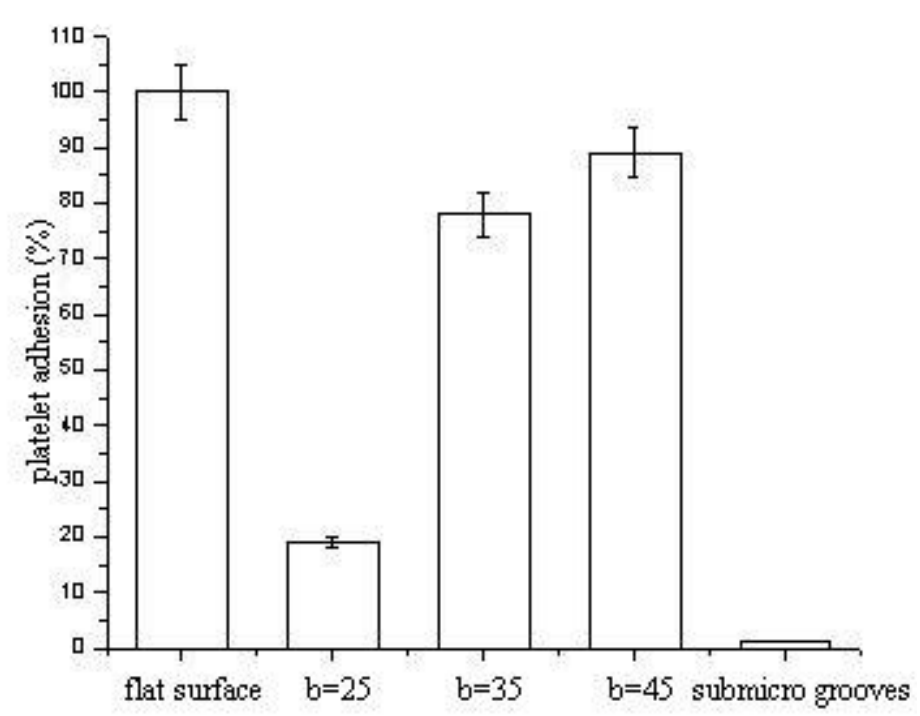

Fig. 5 Platelet adhesions on different surfaces. The values are given as a percentage of adsorption onto flat surface (The error bar is for standard deviation, $n=5$ ). $b$ is the micro pillars' spacing.

The extent of adhesion and coagulation of platelet were observed by SEM. Figure 6 shows micrographs of adhered platelet on different surfaces. Figure 6(a) shows that the morphology of platelets attached on flat surface, spreading flatten and aggregated. As shown in figure 5 as well as in SEM micrographs platelet adhesion has a maximum on the flat surface. On arrays of micro-pillars surfaces, it has a less platelet adhesion as is shown in figure 6(b, c, and d), especially when the surface is super hydrophobic (contact angle $>150^{\circ}$ ) showed in figure 6(b), the adhered number dramatically decreased. Moreover, when the arrays of pillars surface is more hydrophobic, the number of adhesive platelets is less. And interestingly, for patterned nano-grooves surface, it is clear that platelet kept in a normal shape; no significant pseudopodium and aggregation were found. On the nano patterned and hydrophobic surfaces, the shape of most adhered platelets displayed 
regularly and they did not flatten, which indicates that the blood compatibility of the textured fabrics is improved compared with that of flat surface.
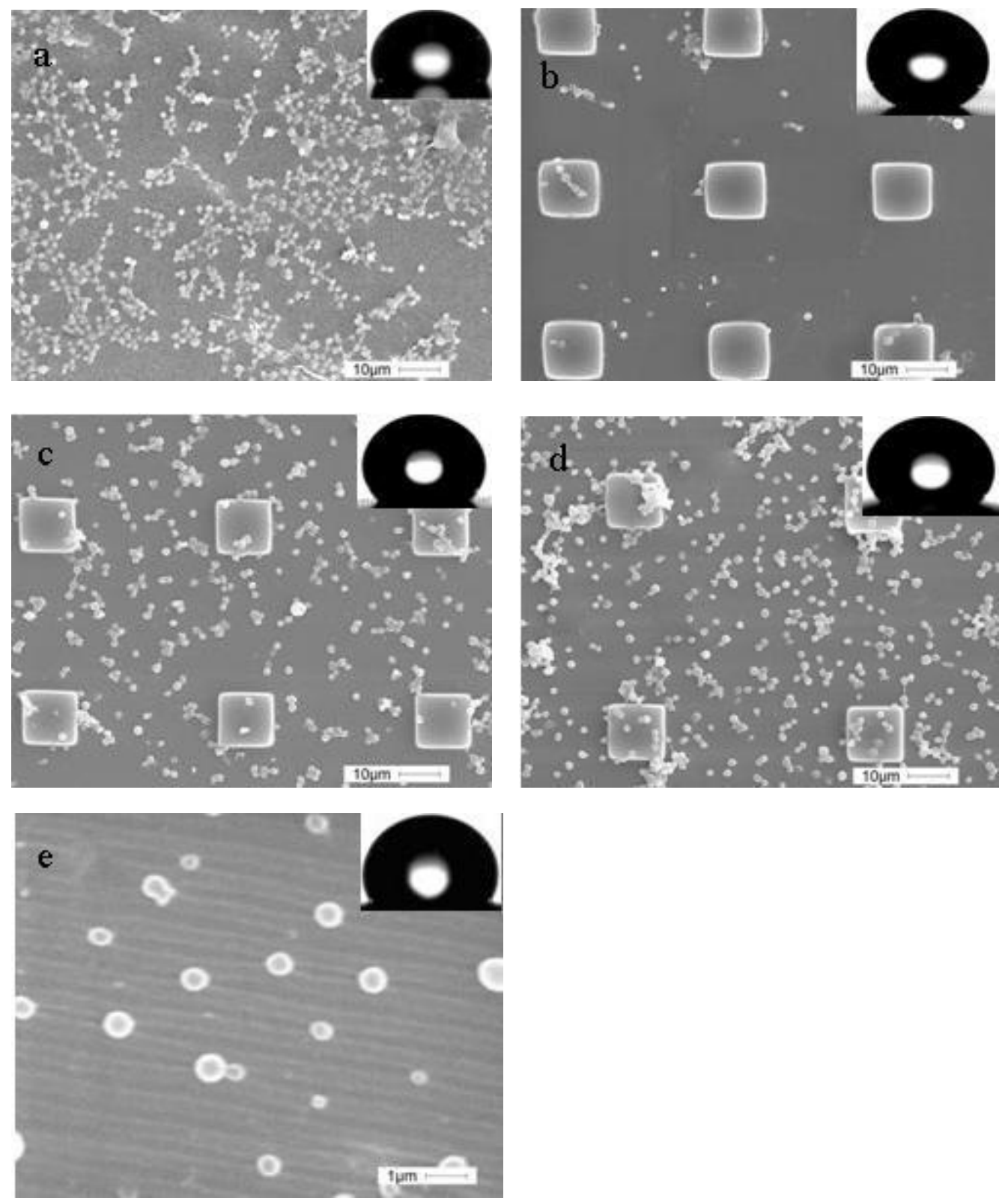

Fig. 6 SEM of platelets adhesion to (a) a flat substrate, (b, c, d) the surfaces with micro-pillars (width $\mathrm{a}=10 \mu \mathrm{m}$, height $\mathrm{h}=5 \mu \mathrm{m}$, and spacing $\mathrm{b}=25 \mu \mathrm{m}, 35 \mu \mathrm{m}, 45 \mu \mathrm{m}$ respectively) and (e) the surface with sub-micro grooves (inset image of contact angle on such surfaces).

Why does the platelet respond these textured structures in this way? One possibility is that the different hydrophobe is affected in some way in the regions. The surface wettability is different on these fabrics, although the chemical compositions of them are the same. Under the action of surface tension, the adhered platelets are activated. They react with artificial surface, aggregate together and release coagulation factors. The surface tension in an area formed into patterned fabrics is different from that of a flat surface, and also micro-pillars with different size have different wettability which is characterized by contact angles. The surface of $156^{\circ}$ contact angle is superhydrophobic; the number of adsorbed platelets is less.

The nano-grooves are interesting and vagarious; it is more desirable to reduce platelet adhesion than that of other patterned surfaces. Moreover, platelets aligned along the grooves. One possibility is that the small spacing between the grooves with the depth of $200 \mathrm{~nm}$ or less inhibits the formation 
of focal contact and prohibits platelet aggregation and pseudopodium formation. It is important and interesting to find the minimum distance for non-adhesion in the future investigation.

\section{Conclusion}

The soft lithography provides a both simple and inexpensive technique to fabricate evenly patterned surfaces, for the research of the effect of surface roughness and hydrophobic property on blood compatibility. On hydrophobic or sub-micro patterned surface, the number of the adhered platelet is less than that of flat surface, and less platelet distorted with pseudopodia. They also keep a normal shape without significant pseudopodium and aggregation. These patterned surfaces display excellent blood compatibility.

The investigation results indicate that both surface wettability and surface morphology play important roles in hemocompatibility. An excellent anticoagulation material should have an ultrahydrophobic merit in order to adsorb platelets as less as possible. Meanwhile, it should also have a relatively fine structure. In this way, the coagulation reaction can be delayed.

\section{Acknowledgements}

This research is supported by the National Natural Science foundation of China (50435030), the Program for New Century Excellent Talents in Chinese University and Excellent Young Scholars foundation of Jiangsu (BK 200607).

\section{References}

[1] L. Hao, J. Lawrence, The adsorption of human serum albumin (HSA) on CO2 laser modified magnesia partially stabilised zirconia (MgO-PSZ), Colloids surf. B Biointerfaces 34 (2004) 87-94

[2] L. Hao, J. Lawrence, L. Li, The wettability modification of bio-grade stainless steel in contact with simulated physiological liquids by the means of laser irradiation, Appl. Surf. Sci. 247 (2005) 453-457

[3] J.C.Stendahl, L. Li, R.C. Claussen and S.I. Stupp, Modification of fibrous poly(L-lactic acid) scaffolds with self-assembling triblock molecules, Biomaterials 25(2004) 5847-5856

[4] P. Favia , L.C. Lopez, E. Sardella , R. Gristina , M. Nardulli , R. d'Agostino Sardella, Low temperature plasma processes for biomedical applications and membrane processing, Desalination 199 (2006) 268-270

[5] P.K. Chu, J. Y. Chen, L. P. Wang and N. Huang, Plasma-surface modification of biomaterials, Mater. Sci. Eng. R Rep. 36 (2002) 143-206

[6] R. Thull, Physicochemical principles of tissue material interactions, Biomol Eng. 19(2002) 43-50

[7] Å. Rosengren, S. Oscarsson, E. Pavlovic, A. Krajewski, A. Ravaglioli and A.Piancastelli, Plasma protein adsorption pattern on characterized ceramic biomaterials, Biomaterials 23 (2002) 1237-1247

[8] Jun Zhou, Jiang Yun, XiaoPeng Zang, Jian Shen and SiCong Lin, Platelet adhesion and protein adsorption on silicone rubber surface by ozone-induced grafted polymerization with carboxybetaine monomer, Colloids surf. B Biointerfaces 41 (2005) 55-62 
[9]. Sunny C.H. Kwok, Jin Wang and Paul K. Chu, Surface energy, wettability, and blood compatibility phosphorus doped diamond-like carbon films, Diamond \& Related Materials 14 (2005) 78-85

[10] B. D. Gates, Qiaobing Xu, J. C. Love, D.B. Wolfe and G.M. Whitesides, unconventional nanofabrication, Annu. Rev. Mater. Res. 34 (2004) 339-372

[11] Heungsoo Shin, Fabrication methods of an engineered microenvironment for analysis of cell-biomaterial interaction, Biomaterials 28 (2007) 126-133

[12] A. Khademhosseini, L. Ferreira, J. Blumling III, J. Yeh, J.M. Karp, J.J. Fukuda and R. Langer, Co-culture of human embryonic stem cells with murine embryonic fibroblasts on microwell-patterned substrates, Biomaterials 27 (2006) 5968-5977

[13] C. Neinhuis and W. Barthlott, Characterization and Distribution of Water-repellent, Self-cleaning Plant Surfaces, Annals of Botany 79 (1997) 667-677

[14] K. A. Mowery, M. H. Schoenfisch, J. E. Saavedra, L. K. Keefer and M. E. Meyerhoff, Preparation and characterization of hydrophobic polymeric films that are thromboresistant via nitric oxide release, Biomaterials 21 (2000) 9-21

[15] S. Sagnella, K. Mai-Ngam, Chitosan based surfactant polymers designed to improve blood compatibility on biomaterials, Colloids surf. B Biointerfaces 42 (2005) 147-155

[16] T. Okada and Y. Ikada, Modification of silicone surface by graft polymerization of acrylamide with corona discharge, Makromol. Chem. 192 (1991) 1705-1713 